\title{
Risk Factors and Psychological Analysis of Chronic Immune Thrombocytopenia in Children
}

This article was published in the following Dove Press journal:

International Journal of General Medicine

Ying Sun

Sili Long

Wenjun Liu

Department of Pediatrics, Affiliated Hospital of Southwest Medical University, Luzhou, Sichuan, People's Republic of China
Correspondence: Wenjun Liu

Department of Pediatrics, Affiliated

Hospital of Southwest Medical University,

Luzhou, Sichuan, People's Republic of

China

Email wenjun_liu@swmu.edu.cn
Objective: To study the risk factors and psychological changes in children with chronic immune thrombocytopenia (CITP).

Methods: This was a retrospective analysis of 234 children with ITP diagnosed and treated in the Affiliated Hospital of Southwest Medical University from January 2016 to December 2018. The clinical characteristics and related laboratory factors of all patients were collected, and their impact on the chronicity of ITP was analyzed. The Eysenck Personality Questionnaire-Children's Version (7-15 years old) is a psychological assessment of children with chronic ITP (CITP).

Results: Univariate analysis found that the course of the initial diagnosis $(\chi 2=6.879$, $\mathrm{P}<0.05)$, the age of onset $(\chi 2=13.846, \mathrm{P}<0.05)$ and the absolute value of the initial diagnosis of peripheral blood lymphocytes $\left(\chi^{2}=6.436, \mathrm{P}<0.05\right)$ had statistical differences in the chronicity of ITP in children; a multivariate analysis of the course of the first diagnosis, the age of onset and absolute lymphocyte count (ALC) revealed that ALC is an independent factor in children's chronic ITP $(\mathrm{P}<0.05)$. Compared with normal children, children with CITP had statistical significance in extraversion $(\mathrm{t}=-3.476, \mathrm{P}<0.05)$; compared with newly diagnosed children, they had statistical significance in extraversion (E) and lying (L) $(\mathrm{P}<0.05)$; and there was no statistical difference compared with persistent children $(\mathrm{P}>0.05)$.

Conclusion: The age of onset, the absolute value of lymphocytes at the initial diagnosis, and the course of the initial diagnosis are important for predicting the chronicity of ITP in children. ALC is an independent predictor; children with chronic ITP have psychological problems.

Keywords: children, chronic immune thrombocytopenia, psychology, risk factors

\section{Introduction}

Childhood immune thrombocytopenia is an acquired autoimmune disease characterized by a decrease in platelet count $\left(<100 \times 10^{9} / \mathrm{L}\right)$ and an increased risk of bleeding. ${ }^{1,2}$ This thrombocytopenia may be due to reduced platelet production and immune-mediated destruction, but usually involves glycoprotein autoantibodies expressed on megakaryocytes. ${ }^{3-5}$ ITP is the most common hemorrhagic disease in children, accounting for one-third of hemorrhagic diseases, ${ }^{6}$ Its onset is usually acute and short-lived, with an incidence of approximately (2 5)/10 million. ${ }^{7,8}$ Under normal circumstances, the symptoms of ITP may be different, but it is often the symptoms of thrombocytopenia, such as mucosal bleeding such as ecchymosis, purpura, and epistaxis. In severe cases, it can cause intracranial hemorrhage., 9,10 The prognosis of children with ITP is good, 80 90\% get remission within 12 months, and 10 20\% progress to chronic ITP. The pathogenesis of CITP is not yet fully understood. It is mostly manifested as prolonged 
disease course, recurrent attacks, and difficult treatment; ${ }^{11,12}$ and CITP also has a certain impact on children's emotions, psychology, and behavior. ${ }^{13}$ The Eysenck Personality Questionnaire (EPQ)-Children's version is divided into four dimensions: psychoticism (P), extraversion (E), neuroticism $(\mathrm{N})$, and lying (L). It involves fewer concepts and is convenient to test. With good reliability and validity, it is one of the most influential psychological scales in the world. ${ }^{14,15}$ This study retrospectively analyzed the clinical data of 234 children with primary ITP in our hospital, explored the risk factors and psychological conditions of CITP, and provided a certain basis for clinical treatment.

\section{Patients and Methods}

\section{General Information}

A retrospective analysis of 234 children with ITP diagnosed in the Affiliated Hospital of Southwest Medical University from January 2016 to December 2018, including 143 males and 91 females, aged from January to 156 months, 181 cases in rural areas and 53 cases in cities, 153 cases were newly diagnosed, 47 cases were persistent, and 34 cases were chronic. The diagnosis and staging standards of the disease should refer to the National Health Commission's "Diagnosis and treatment guideline of primary immune thrombocytopenia in children (2019 edition)" (11 (hereinafter referred to as the "standards"). Inclusion criteria: in line with the diagnostic criteria of the "Standards": (1)At least two blood tests indicate thrombocytopenia and no abnormal blood cell morphology; (2) The spleen is generally not enlarged; the number of megakaryocytes in the bone marrow examination is normal or increased, accompanied by maturation disorders; (4)Exclude yourself Secondary thrombocytopenia such as immune diseases, drug-induced, infection, myelodysplasia, and hematological malignancies. Stages of the disease: (1)Newly diagnosed ITP: children within 3 months after diagnosis; (2)Persistent ITP: children with a continuous decrease in platelets from 3 to 12 months after diagnosis and who cannot maintain complete remission after stopping treatment; (3)chronic ITP: Children with thrombocytopenia lasting more than 12 months. As of December 2019, there are 32 children with persistent ITP aged $\geq 7$ years, 51 children with newly diagnosed ITP aged $\geq 7$ years, and 24 children with chronic ITP aged $\geq 7$ years. Personality Questionnaire for children (7-15 years old) for psychological assessment.

\section{Main Research Indicators}

Collecting all children's clinical characteristics (age of onset, gender, degree of bleeding, first diagnosis, etc), laboratory data (initial platelet count, newly diagnosed ALC, platelet antibodies, megakaryocyte count, viral infection, etc). Treatment methods (hormones (eg prednisone), immunoglobulin, immunoglobulinandhormones, other).

Children's bleeding degree classification, ${ }^{16}$ asymptomatic: Accidentally found that the PLT count decreased; mild: petechiae, ecchymosis and other skin manifestations, occasionally minor nose bleeding, which does not interfere with normal life; moderate: more severe skin manifestations, mucosal lesions, Severe epistaxis; severe: epistaxis, black stool and other bleeding events require

Table I Comparison of Gender and Age Between Children with Chronic ITP and Children with Non-Chronic ITP

\begin{tabular}{|c|c|c|c|c|c|c|}
\hline & \multirow[b]{2}{*}{ Total } & \multicolumn{2}{|c|}{ Gender } & \multicolumn{3}{|c|}{ Age(Year) } \\
\hline & & Male & Female & $\leq \mathbf{I}$ & $1-7$ & $\geq 7$ \\
\hline CITP & 34 & 16 & 18 & 4 & 21 & 9 \\
\hline NCITP & 200 & 127 & 73 & 84 & 65 & 51 \\
\hline$\chi^{2}$ & \multicolumn{4}{|l|}{3.305} & \multicolumn{2}{|c|}{13.846} \\
\hline$P$ value & \multicolumn{4}{|l|}{0.069} & \multicolumn{2}{|c|}{0.001} \\
\hline
\end{tabular}

Table 2 Comparison of the Degree of Bleeding and the Course of the Initial Diagnosis Between Children with Chronic ITP and Children with Non-Chronic ITP

\begin{tabular}{|l|l|l|l|l|l|l|}
\hline \multirow{2}{*}{} & \multicolumn{2}{|l|}{ Degree of Bleeding } & \multicolumn{2}{l|}{ Initial Diagnosis(Month) } \\
\cline { 2 - 7 } & Total & Asymptomatic and Mild & Moderate & Severe & $<1$ & $\geq$ I \\
\hline $\begin{array}{l}\text { CITP } \\
\text { NCITP }\end{array}$ & 34 & 7 & 17 & 10 & 26 & 8 \\
\hline$\chi^{2}$ & 200 & 70 & 92 & 38 & 183 & 6.879 \\
\hline P value & 3.470 & 0.176 & & 0.009 \\
\hline
\end{tabular}


hospitalization or blood transfusion, seriously affect the quality of life of children and even threaten life.

The Eysenck Personality Questionnaire (EPQ) has 88 questions. The subjects answer "yes" or "no" according to their actual situation to obtain a rough score of the total score of each dimension. The rough score is converted into a $\mathrm{T}$ score according to the $\mathrm{T}$ score table ( $\mathrm{T}$ score between 43.3 and 56.7 points is intermediate type; T score between 38.5 and 43.3 points or between 56.7 and 61.5 points is prone type; $\mathrm{T}$ score is below 38.5 points or above 61.5 points is typical). The personality characteristics of the testees can be analyzed. ${ }^{14,15}$

\section{Treatment Methods}

According to the guidelines of the "Specifications", when PLT $<20 \times 10^{9} / \mathrm{L}$ and accompanied by bleeding, using prednisone $1.5 \sim 2.0 \mathrm{mg} \cdot \mathrm{kg}^{-1} \cdot \mathrm{d}^{-1}$ (starting maximum dose $\leq 60 \mathrm{mg} / \mathrm{d}$ ), or intravenous infusion of gamma globulin $0.4 \mathrm{~g} \cdot \mathrm{kg}^{-1} \cdot \mathrm{d}^{-1} \times \quad(3 \sim 5) \quad \mathrm{d}$. Close observation when PLT $\geq 20 \times 10^{9} / \mathrm{L}$ and no active bleeding. When PLT $<10 \times 10^{9} / \mathrm{L}$ and other systemic bleeding occurs, using gamma globulin $1.0 \mathrm{~g} \cdot \mathrm{kg}^{-1} \cdot \mathrm{d}^{-1} \times(2 \sim 3) \mathrm{d}$ or high-dose hormone shock, and at the same time, appropriate platelet transfusions make PLT $>50 \times 10^{9} / \mathrm{L}$. For children with ITP who need second-line treatment, thrombopoietic drugs (recombinant human thrombopoietin, ${ }^{17}$ Eltrombopag, ${ }^{18,19}$ etc), CD20 monoclonal antibody (rituximab), ${ }^{20}$ even vincristine, azathioprine, cyclosporine A and other immunosuppressive agents ${ }^{21,22}$ can be selected as appropriate.

\section{Follow-Up}

After admission, the child's information will be collected, and the child's family members will be notified of the precautions after discharge. Regular outpatient review, record changes in the child's condition, telephone follow-up and conduct EPQ questionnaire surveys. The follow-up period ends in December 2019. All children have been followed up. And the family members of the children had informed consent to the treatment plan. The study was also approved by the Ethics Committee of the affiliated Hospital of Southwest Medical University. The Affiliated Hospital of Southwest Medical University is a comprehensive hospital integrating medical treatment, teaching, and scientific research. Patient parental consent to review their medical records was not required by the Ethics Committee of the Affiliated Hospital of Southwest Medical University. Because the patient's interests are not compromised and their privacy is protected, this is the reason for the waiver. We keep all patient data strictly confidential and comply with the Declaration of Helsinki.

Table 3 Comparison of PLT Antibodies, PLT Counts and Newly Diagnosed Megakaryocytes in Children with Chronic ITP and NonChronic ITP Children

\begin{tabular}{|c|c|c|c|c|c|c|c|c|}
\hline & \multirow[b]{2}{*}{ Total } & \multicolumn{2}{|c|}{ PLT Antibody } & \multicolumn{3}{|c|}{ PLT Count $\left(\times 10^{9} / \mathrm{L}\right)$} & \multicolumn{2}{|c|}{ Megakaryocyte Count } \\
\hline & & Negative & Positive & $\leq 10$ & $10-20$ & $\geq 20$ & Increase & Normal \\
\hline CITP & 34 & 30 & 4 & 17 & 9 & 8 & 29 & 5 \\
\hline NCITP & 200 & 172 & 28 & 97 & 39 & 64 & 164 & 36 \\
\hline$\chi^{2}$ & \multicolumn{3}{|l|}{0.123} & \multicolumn{3}{|c|}{1.379} & \multicolumn{2}{|l|}{0.218} \\
\hline$P$ value & \multicolumn{3}{|l|}{0.726} & \multicolumn{3}{|c|}{0.502} & \multicolumn{2}{|l|}{0.640} \\
\hline
\end{tabular}

Table 4 Comparison of Cytomegalovirus Infection and Newly Diagnosed ALC in Children with Chronic ITP and Non-Chronic ITP Children

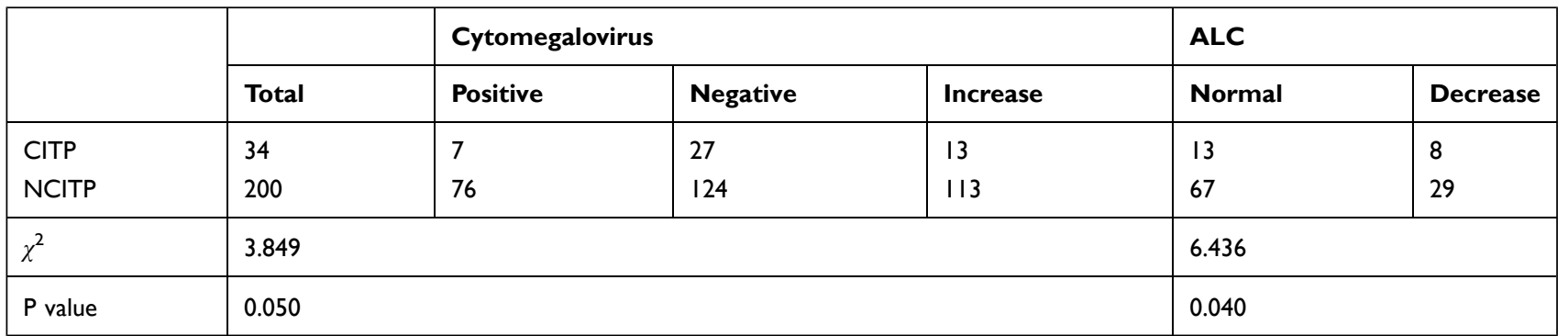




\section{Statistical Analysis}

Statistical software SPSS16.0 was used for data analysis. Measurement data were expressed as mean \pm standard deviation $( \pm \mathrm{s})$. The $\chi^{2}$ test was used to analyze the factors affecting the chronicity and effectiveness of ITP. Logistic regression analysis was performed on the age of onset, the course of first diagnosis and the first diagnosis of ALC. Psychological evaluation adopts the $t$-test of the mean of two samples in a group design, with the test standard of $\alpha=0.05$, and $\mathrm{P}<0.05$ indicates that the difference is statistically analyzed.

\section{Results}

Comparison of Gender and Age Between Children with Chronic ITP and Children with Non-Chronic ITP

Among 234 children with ITP, 143 were males and 91 were females, aged from 1 month to 156 months. There was no statistical significance in gender between children with chronic ITP and children with non-chronic ITP $\left(\chi^{2}=3.305, \mathrm{P}=0.069\right)$. The age of onset of chronic ITP children is mostly in 1-7 years old, non-chronic ITP occurs most often in those younger than 1 year old. The age of chronic ITP children and non-chronic ITP children is statistically significant $\left(\chi^{2}=13.846, \mathrm{P}=0.001\right)$ (Table 1).

\section{Comparison of the Degree of Bleeding} and the Course of Initial Diagnosis Between Children with Chronic ITP and Children with Non-Chronic ITP

Among the 234 children with ITP, 77 cases were asymptomatic and mild bleeding, 109 cases were moderate, and 48 cases were severe. There was no statistical significance in the degree of bleeding between children with chronic ITP and children with nonchronic ITP $\left(\chi^{2}=3.470, \mathrm{P}=0.176\right)$. There were 209 cases with the initial diagnosis course of $<1$ month and 25 cases $\geq 1$ month. The initial diagnosis of children with chronic ITP and non-chronic ITP were mainly concentrated within one month, and there was a statistical difference between the two in the initial diagnosis $\left(\chi^{2}=6.879, \mathrm{P}=0.009\right)$ (Table 2).

\section{Comparison of PLT Antibody and PLT Counts Between Children with Chronic ITP and Children with Non-Chronic ITP}

Among the 234 children with ITP, 202 were PLT antibody negative and 32 were positive; 72 cases were PLT $\geq 20 \times 10^{9} \mathrm{~L}$ at first diagnosis, 114 cases were PLT $<10 \times 10^{9} \mathrm{~L} 48$ cases were $(10 \sim 20) \times 10^{9} \mathrm{~L}$, and the number of megakaryocytes at first diagnosis increased 193 cases, 41 normal cases. The results of the study showed that children

Table 5 Comparison of Treatment Methods Between Children with Chronic ITP and Children with Non-Chronic ITP

\begin{tabular}{|c|c|c|c|c|c|}
\hline & \multicolumn{5}{|c|}{ Treatment } \\
\hline & Total & Immunoglobulin & Hormones & Immunoglobulin \& Hormones & Other \\
\hline CITP & 34 & 2 & 1 & 24 & 7 \\
\hline NCITP & 200 & 20 & 8 & 132 & 40 \\
\hline$\chi^{2}$ & \multicolumn{5}{|l|}{0.706} \\
\hline$P$ value & \multicolumn{5}{|l|}{0.872} \\
\hline
\end{tabular}

Table 6 The Relationship Between Age, First-Diagnosed Disease Course and First-Diagnosed ALC and Chronic ITP

\begin{tabular}{|l|l|l|l|l|l|l|l|}
\hline Index & Estimate & Standard Error & Wald & DF & P & \multicolumn{2}{|l|}{ 95\% Confidence Interval } \\
\cline { 4 - 8 } & & & & & & Upper Limit & Lower Limit \\
\hline Age & -0.005 & 0.005 & 0.889 & 1 & 0.346 & 0.986 & 1.005 \\
Initial diagnosis & 0.003 & 0.002 & 1.060 & 1 & 0.303 & 0.998 & 1.007 \\
ALC & -0.443 & 0.129 & 11.825 & 1 & 0.001 & 0.499 & 0.826 \\
\hline
\end{tabular}


with chronic ITP and non-chronic ITP children had PLT antibodies $\left(\chi^{2}=0.123, \quad \mathrm{P}=0.726\right)$, PLT count $\quad\left(\chi^{2}=1.379\right.$, $\mathrm{P}=0.502$ ), and the number of newly diagnosed megakaryocytes $\left(\chi^{2}=0.218, \mathrm{P}=0.640\right)$ has no statistical significance (Table 3).

\section{Comparison of Cytomegalovirus Infection and Newly Diagnosed ALC in Children with Chronic ITP and Non-Chronic ITP Children}

Among the 234 children with ITP, 83 were infected with cytomegalovirus. There was no statistically significant difference between children with chronic ITP and children with non-chronic ITP $\left(\chi_{2}=3.849, \mathrm{P}=0.050\right)$. The newly diagnosed ALC increased by 126 cases, the normal 80 cases, and the decreased 28 cases. The results of the study showed that children with chronic ITP and nonchronic ITP have statistical significance in newly diagnosed ALC $\left(\chi^{2}=6.436, \mathrm{P}=0.040\right)$ (Table 4).

\section{Comparison of Treatment Methods Between Children with Chronic ITP and Children with Non-Chronic ITP}

Among the 234 children with ITP, 22 were treated with immunoglobulin, 9 with hormones, 156 with immunoglobulin and hormones, and the other 47 ( 2 of which were transfused with platelets due to intracranial hemorrhage). The four treatment methods are mostly gamma globulin combined with glucocorticoids. Statistical analysis found that there is no significant difference in the treatment methods between children with chronic ITP and children with non-chronic ITP $(\chi 2=0.706, \mathrm{P}=0.872)$ (Table 5).

\section{The Relationship Between Age,} First-Diagnosed Disease Course and First-Diagnosed ALC and Chronic ITP

Logistic regression analysis was performed on the age of onset, the course of the initial diagnosis, and the initial

\section{ROC Curve}

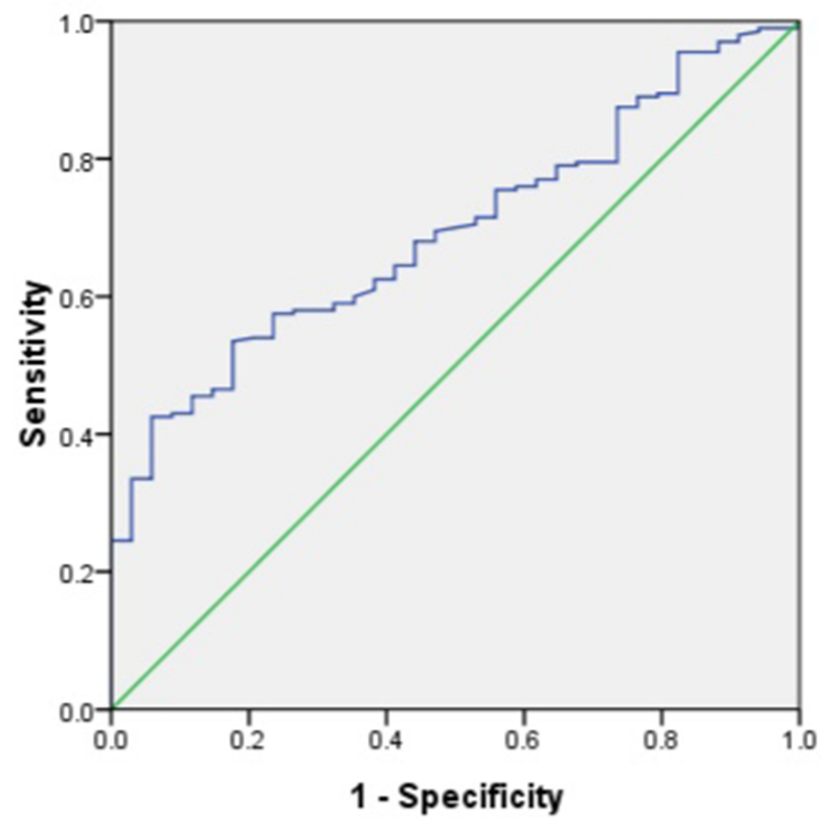

Figure I The area under the curve for the first diagnosed ALC to predict ITP.

diagnosis of ALC, and it was found that the initial diagnosis of ALC was an independent influencing factor of chronic ITP (Table 6). The ROC curve was drawn with newly diagnosed ALC as the test variable and chronic as the state variable. When the critical value ALC is $4.905 \times 10^{9} \mathrm{~L}$, the area under the curve is 0.694 , the sensitivity is $42.5 \%$, and the specificity is $94.1 \%(\mathrm{P}<0.05)$ (Figure 1). It is suggested that when $\operatorname{ALC}<4.905 \times 10^{9} \mathrm{~L}$, the child is prone to develop chronic ITP.

\section{EPQ Psychological Evaluation of Children with Chronic ITP and Normal Children}

The EPQ psychological evaluation of children with chronic ITP has a lower score on extraversion (38.54 $\pm 3.89)$ than that of normal children $(43.04 \pm 5.01)$, which is statistically significant $(\mathrm{P}<0.05)$, indicating that chronic disease causes children with quiet, emotionally stable, and introverted personality characteristics. In terms of

Table 7 EPQ Psychological Evaluation Analysis of Children with Chronic ITP and Normal Children

\begin{tabular}{|l|l|l|l|l|}
\hline & P & E & N & L \\
\hline CITP $(n=24)$ & $46.17 \pm 3.60$ & $38.54 \pm 3.89$ & $47.92 \pm 5.16$ & $53.92 \pm 6.39$ \\
Normal child $(n=24)$ & $45.54 \pm 3.45$ & $43.04 \pm 5.01$ & $46.75 \pm 5.45$ & $52.79 \pm 4.47$ \\
t & 0.614 & -3.476 & 0.762 & 0.706 \\
P & 0.542 & 0.001 & 0.450 & 0.484 \\
\hline
\end{tabular}




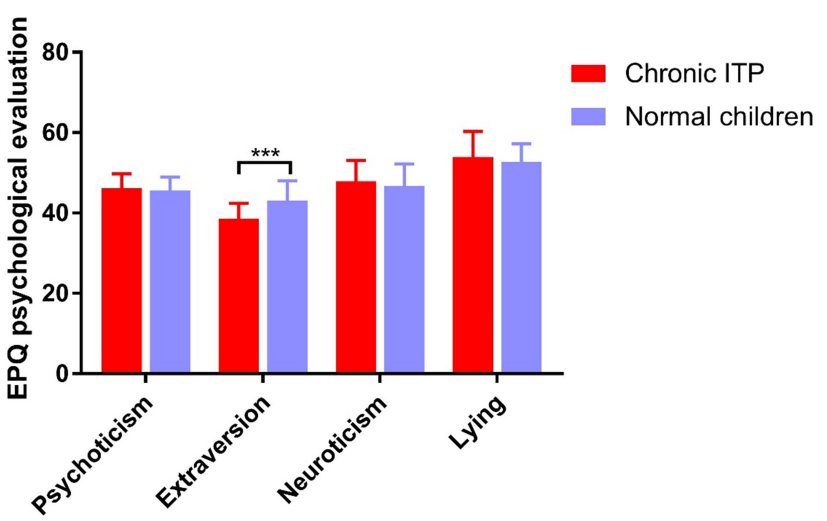

Figure 2 EPQ psychological evaluation of children with chronic ITP and normal children $(* * * \mathrm{p}<0.05)$.

psychoticism (P), neuroticism $(\mathrm{N})$ and lying $(\mathrm{L})$, the two groups of patients were not statistically significant $(\mathrm{P}>0.05)$. (Table 7, Figure 2).

\section{EPQ Psychological Evaluation of Children with Chronic ITP and Newly Diagnosed Children with ITP}

In terms of extraversion, children with chronic ITP (38.54 \pm 3.89 ) have lower scores than children with newly diagnosed ITP (41.98 \pm 4.52$)$, which is statistically significant $(\mathrm{P}<0.05)$, reflecting the introverted personality characteristics of children with chronic diseases. In terms of lying, the scores of children with chronic ITP (53.92 \pm 6.39$)$ are higher than those with newly diagnosed ITP $(50.31 \pm 5.21)$, and the difference is statistically significant $(\mathrm{P}<0.05)$, suggesting that chronic ITP children are more sensitive and unwilling to show their true self. In psychoticism and neuroticism, there was no statistical significance in children with chronic ITP and newly diagnosed ITP $(\mathrm{P}>0.05)$. (Table 8, Figure 3).

\section{EPQ Psychological Evaluation of Children with Chronic ITP and Children with Persistent ITP}

In the EPQ psychological evaluation comparison of children with chronic ITP and persistent ITP, there was no statistical significance in psychoticism $(\mathrm{P})$, extraversion $(\mathrm{E})$, neuroticism $(\mathrm{N})$, and lying $(\mathrm{L})(\mathrm{P}>0.05)$. (Table 9, Figure 4).

\section{Discussion}

ITP is the most common bleeding disorder in children, with a good prognosis, but $10 \%$ to $20 \%$ still develop CITP. CITP relapses and seriously affects the quality of life of children. ${ }^{23,24}$ The pathogenesis of ITP is not yet fully understood. It is mainly related to humoral and cellular immune dysfunction. Recent studies have found that Fc $\gamma$ receptor-independent platelet clearance pathways, abnormal miRNAs of genetic factors, and infection factors are related to CITP. ${ }^{25-27}$ Some studies have shown that factors such as the course of first diagnosis, age of onset, history of infection or vaccination, number of newly diagnosed platelets, and degree of bleeding are related to the poor prognosis of children with ITP. However, whether viral infection, lymphocyte count at first diagnosis, antiplatelet antibody, megakaryocyte count and other factors are related to prognosis, there are currently different opinions in domestic and foreign research. ${ }^{28-31}$

The newly diagnosed ALC is related to the immunity of the child. Lyu et $\mathrm{al}^{32}$ showed that the absolute value of newly diagnosed lymphocytes is an independent factor leading to chronic ITP in children. When ALC $3.005 \times 10^{9} / \mathrm{L}$ was used as the cutoff value, it was found that the non-remission rate was statistically different. Grouped by age 5 , the remission rate between different age groups was found to be statistically significant, which is consistent with our research results. Different age groups and absolute values of newly diagnosed lymphocytes have statistical significance for chronic ITP. Wang Yongxin et $\mathrm{al}^{33}$ showed that chronic ITP and non-chronic ITP are statistically significant in the course of the initial diagnosis and the initial diagnosis of ALC. The course of the initial diagnosis of chronic ITP children is longer than non-chronic ITP children, and the newly diagnosed ALC is less than non-chronic ITP children. It is believed that the initial diagnosis of the disease is a risk factor for chronic ITP, and the initial diagnosis of ALC is a protective factor

Table 8 EPQ Psychological Evaluation of Children with Chronic ITP and Newly Diagnosed Children with ITP

\begin{tabular}{|l|l|l|l|l|}
\hline & P & E & N & L \\
\hline CITP $(n=24)$ & $46.17 \pm 3.60$ & $38.54 \pm 3.89$ & $47.92 \pm 5.16$ & $53.92 \pm 6.39$ \\
Newly diagnosed ITP $(n=5 I)$ & $45.00 \pm 2.47$ & $41.98 \pm 4.52$ & $45.96 \pm 4.20$ & $50.31 \pm 5.21$ \\
t & 1.438 & -3.208 & 1.747 & 2.596 \\
P & 0.160 & 0.002 & 0.085 & 0.011 \\
\hline
\end{tabular}




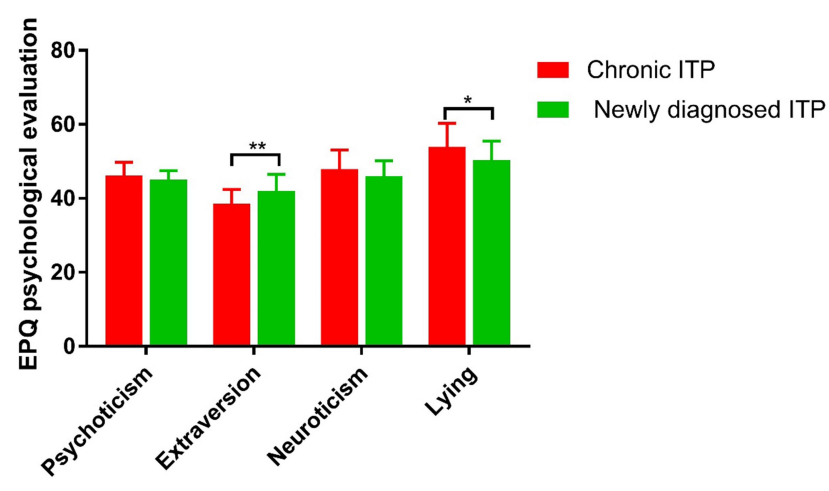

Figure 3 EPQ psychological evaluation of children with chronic ITP and newly diagnosed children with ITP $\left(* * p<0.05, *^{*}<0.05\right)$.

for chronic ITP. This is consistent with the results of our study. Logistic regression analysis found that when ALC $>4.905 \times 10^{9} / \mathrm{L}$, the possibility of children's ITP progressing to chronic ITP is very low, and ALC is an independent predictor of chronic ITP.

Some studies ${ }^{30,34}$ have found that factors affecting chronic ITP include the age of onset, gender, initial platelet count, and infection. Kalfon et $\mathrm{al}^{30}$ pointed out that children with acute ITP are more likely to have skin bleeding than chronic children. The combination of different types of skin bleeding reduces the risk of chronic ITP in children, and age is the most important risk factor for chronic ITP in children. Acute ITP is a self-limiting illness often occurring after an infectious disease, platelet counts $<20 \times 10^{9} / \mathrm{L}$ in most affected children, some guidelines aimed at standardizing acute ITP diagnosis and treatment by using a bleeding score. ${ }^{35}$ Ahmed et $\mathrm{al}^{36}$ found that when $\mathrm{ALC}<3.050 \times 10^{9} / \mathrm{L}$, there is a significant risk of developing chronic ITP, and their research results indicate that ALC is a strong predictor of chronic ITP. In this study, the predictive factors of chronic ITP include the age of onset, first-diagnosed disease course and initial ALC, while other factors (gender, initial platelet count, infection, etc) have no significant statistical significance in predicting chronic ITP.

During the long-term treatment of children with chronic ITP, the children and their parents will experience emotional and psychological changes. Children will be upset because of long-term medical examinations, drug use, and in order to avoid bleeding, they cannot participate in activities they love, and cannot play with their classmates. ${ }^{37,38}$ The results of the EPQ questionnaire showed that the extraversion scores of chronic children were significantly lower than those of normal children. Those with high scores are cheerful, lively, easy-going, optimistic, casual, eager for stimulation and adventure, good at communication, and their behavior is easily affected by impulse; Low scorers are quiet, emotionally stable, good at introspection, rigorous and planned, do not like impulse and adventure, except for close friends, generally speaking less indifferent to people and keeping a certain distance, indicating that chronic diseases cause children with introverted psychological characteristics. Wu Jinxia et $\mathrm{al}^{39}$ compared the extraversion scores and lying scores of 70 children with chronic diseases with 70 normal children and concluded that chronic diseases have an important influence on the children's personality, and it is consistent with our results in extraversion. In our study, the extraversion score of children with chronic ITP is lower than that of children with newly diagnosed ITP, which further reflects the introverted personality characteristics of children with chronic ITP. In terms of lying, high scorers have a tendency to cover up, suggesting that long-term illness makes children more sensitive and unwilling to show their true self, thereby covering themselves, while low scorers are simpler and naive. In short, children with chronic ITP have more difficulties in emotion, psychology and executive function during long-term treatment. Therefore, in addition to promoting the increase of platelet count, appropriate psychological intervention and adjustment are of great significance to the physical and mental health of children. ${ }^{13}$

The application of long-term corticosteroids and immunosuppressive agents has serious adverse effects, which have a negative impact on children's physical and mental health, quality of life and family. ${ }^{40}$ Physicians and children need

Table 9 EPQ Psychological Evaluation of Children with Chronic ITP and Children with Persistent ITP

\begin{tabular}{|l|l|l|l|l|}
\hline & P & E & N & L \\
\hline CITP $(n=24)$ & $46.17 \pm 3.60$ & $38.54 \pm 3.89$ & $47.92 \pm 5.16$ & $53.92 \pm 6.39$ \\
Persistent ITP (n=32) & $46.03 \pm 3.23$ & $40.16 \pm 5.47$ & $46.69 \pm 4.77$ & $52.56 \pm 4.33$ \\
t & 0.148 & -1.290 & 0.922 & 0.895 \\
P & 0.883 & 0.202 & 0.361 & 0.376 \\
\hline
\end{tabular}




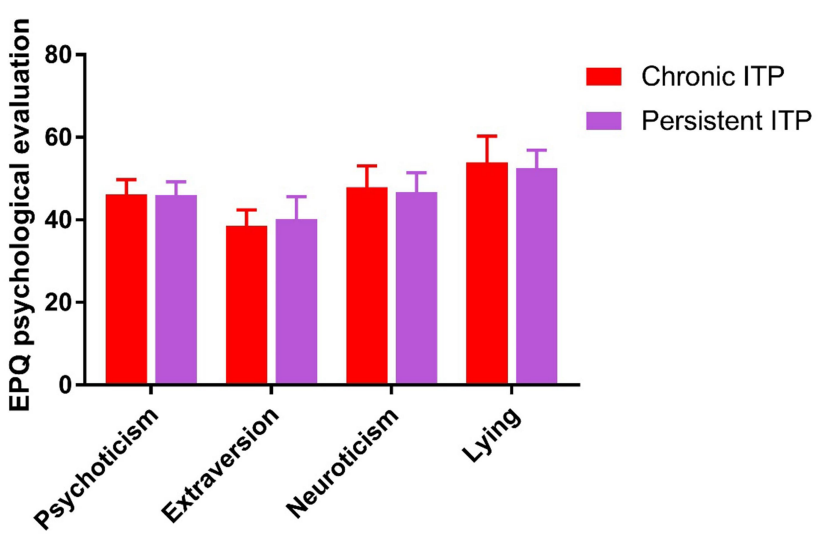

Figure 4 EPQ psychological evaluation of children with chronic ITP and children with persistent ITP.

reliable and instructive conclusions, which will help clinicians to effectively monitor and improve the outcome in the early treatment period, and reduce the impact of the disease on the children's daily life and psychology. Narrative medicine can improve the quality of life of pediatric chronic ITP patients and their families, moreover, it can also quantify the familial burden imposed by the illness. ${ }^{41}$ The risk factors of chronic ITP in children are a hot research topic by scholars at home and abroad. However, research on certain factors is still lacking and there are different opinions. Therefore, we need more comprehensive, reasonable and rigorous research to provide a basis for the clinical judgment of the prognosis of children's ITP and to guide the treatment and management of the disease.

\section{Conclusion}

The age of onset, the absolute value of lymphocytes at the initial diagnosis, and the course of the initial diagnosis are important for predicting the chronicity of ITP in children. ALC is an independent predictor; children with chronic ITP have psychological problems.

\section{Acknowledgments}

We would like to thank all the participants in the studies.

\section{Disclosure}

The authors declare that they have no conflicts of interest.

\section{References}

1. Zhang L, Zhang M, Du X, et al. Safety and efficacy of eltrombopag plus pulsed dexamethasone as first-line therapy for immune thrombocytopenia. $\quad B r \quad J$ Haematol. 2020;189(2):369-378. doi:10.1111/bjh.16327
2. Petrovic D, Benzon B, Batinic M, et al. Hypovitaminosis $\mathrm{D}$ influences the clinical presentation of immune thrombocytopenia in children with newly diagnosed disease. J Clin Med. 2019;8 (11):1861. doi:10.3390/jcm8111861

3. Samson M, Fraser W, Lebowitz D. Treatments for primary immune thrombocytopenia: a review. Cureus. 2019;11(10):e5849. doi: $10.7759 /$ cureus. 5849

4. Kado R, McCune WJ. Treatment of primary and secondary immune thrombocytopenia. Curr Opin Rheumatol. 2019;31(3):213-222. doi:10.1097/BOR.0000000000000599

5. Miltiadous O, Hou M, Bussel JB. Identifying and treating refractory ITP: difficulty in diagnosis and role of combination treatment. Blood. 2020;135(7):472-490. doi:10.1182/blood.2019003599

6. Li H, Yang H, Liu WJ. Efficacy of romiplostim in the treatment of ITP in children: a meta-analysis. Eur Rev Med Pharmacol Sci. 2018;22(18):6162-6169. doi:10.26355/eurrev_201809_15958

7. Yong M, Schoonen WM, Li L, et al. Epidemiology of paediatric immune thrombocytopenia in the general practice research database. $\mathrm{Br} J$ Haematol. 2010;149(6):855-864. doi:10.1111/j.13652141.2010.08176.x

8. Neunert C, Terrell DR, Arnold DM, et al. American society of hematology 2019 guidelines for immune thrombocytopenia. Blood Adv. 2019;3(23):3829-3866. doi:10.1182/bloodadvances.2019000966

9. Liu WJ, Bai J, Guo QL, et al. Role of platelet function and platelet membrane glycoproteins in children with primary immune thrombocytopenia. Mol Med Rep. 2016;14(3):2052-2060. doi:10.3892/mmr.2016.5504

10. Onisâi M, Vlădăreanu AM, Spînu $\mathrm{A}$, et al. Idiopathic Thrombocytopenic Purpura (ITP) - new era for an old disease. Rom J Intern Med. 2019;57(4):273-283. doi:10.2478/rjim-2019-0014

11. National Health Commission. Diagnosis and treatment guideline of primary immune thrombocytopenia in children. Clin Educ Gen Pract. 2019;17(12):1059-1062.

12. Youssef MAM, Salah Eldeen E, Elsayh KI, et al. High dose dexamethasone as an alternative rescue therapy for active bleeding in children with chronic ITP: clinical and immunological effects. Platelets. 2019;30(7):886-892. doi:10.1080/09537104.2018.1530347

13. Towner S, Berger ZE, Titman P, et al. Fatigue, executive function and psychological effects in children with immune thrombocytopenia: across-sectional study. $B r \quad J$ Haematol. 2020;189(3):534-542. doi:10.1111/bjh.16387

14. Huang Y, He M, Li A, Wu K. Personality, behavior characteristics, and life quality impact of children with dyslexia. Int $J$ Environ Res Public Health. 2020;17(4).

15. Liu C, Chen L, Chen S. Influence of neuroticism on depressive symptoms among chinese adolescents: the mediation effects of cognitive emotion regulation strategies. Front Psychiatry. 2020;420.

16. Bolton-Maggs P. Severe bleeding in idiopathic thrombocytopenic purpura. J Pediatr Hematol Oncol. 2003;25:S47-S51. doi:10.1097/ 00043426-200312001-00011

17. Li Y, Wang YY, Fei HR, et al. Efficacy of low-dose rituximab in combination with recombinant human thrombopoietin in treating ITP. Eur Rev Med Pharmacol Sci. 2015;19(9):1583-1588.

18. Kim TO, Despotovic J, Lambert MP. Eltrombopag for use in children with immune thrombocytopenia. Blood Adv. 2018;2(4):454-461. doi:10.1182/bloodadvances.2017010660

19. Wong RSM, Saleh MN, Khelif A, et al. Safety and efficacy of long-term treatment of chronic/persistent ITP with eltrombopag: final results of the EXTEND study. Blood. 2017;130 (23):2527-2536. doi:10.1182/blood-2017-04-748707

20. Liu JX, Zhang HX, Li DP, et al. Curative efficacy of rituximab for ITP patients with different sensitivity to hormone. Zhongguo Shi Yan Xие Ye Xие Za Zhi. 2019;27(5):1602-1606. doi:10.19746/j.cnki. issn.1009-2137.2019.05.037 
21. Kühne T. Diagnosis and management of immune thrombocytopenia in childhood. Hamostaseologie. 2017;37(1):36-44. doi:10.5482/ HAMO-16-06-0017

22. Grace RF, Neunert C, Second-line therapies in immune thrombocytopenia. Hematology Am Soc Hematol Educ Program. 2016;(1):698-706. doi:10.1182/asheducation-2016.1.698

23. Zhang H, Wang L, Quan M, et al. Health-related quality of life in children with chronic immune thrombocytopenia in China. Health Qual Life Outcomes. 2016;14:45. doi:10.1186/s12955-016-0445-3

24. Yahia S, Wahba Y, El-Gilany AH. Psychiatric disorders and quality of life in egyptian patients with chronic immune thrombocytopenic purpura: a single center study. Indian J Hematol Blood Transfus. 2019;35(2):347-351. doi:10.1007/s12288-018-1031-0

25. Swinkels M, Rijkers M, Voorberg J, et al. Emerging concepts in immune thrombocytopenia. Front Immunol. 2018;9:880. doi:10.3389/fimmu.2018.00880

26. Dou X, Yang R. Current and emerging treatments for immune thrombocytopenia. Expert Rev Hematol. 2019;12(9):723-732. doi:10.1080/17474086.2019.1636644

27. Zufferey A, Kapur R, Semple JW. Pathogenesis and therapeutic mechanisms in immune thrombocytopenia (ITP). J Clin Med. 2017;6(2):16. doi:10.3390/jcm6020016

28. Güngör T, Arman Bilir Ö, Koşan V, et al. Retrospective evaluation of children with immune thrombocytopenic purpura and factors contributing to chronicity. Pediatr Neonatol. 2019;60(4):411-416. doi:10.1016/j.pedneo.2018.10.002

29. Almohammadi A, Lundin MS, Abro C, et al. Epistaxis and gross haematuria with severe thrombocytopaenia associated with influenza vaccination. BMJ Case Rep. 2019;12(5):e229423. doi:10.1136/bcr2019-229423

30. Kalfon S, Hamadeh H, Schachter Y, et al. Cutaneous hemorrhage types as supportive factors for predicting chronic immune thrombocytopenia in children. J Pediatr Hematol Oncol. 2018;40(5):337-340. doi:10.1097/MPH.0000000000001167

31. Jung JY, O AR, Kim JK, Park M. Clinical course and prognostic factors of childhood immune thrombocytopenia: single center experience of 10 years. Korean $J$ Pediatr. 2016;59(8):335-340. doi:10.3345/kjp.2016.59.8.335
32. Lyu ME, Li Y, Liu WJ, et al. Relationship between initial absolute lymphocyte counts and the prognosis of children with primary immune thrombocytopenia. $J$ Appl Clin Pediatr. 2015;30 (15):1147-1151.

33. Wang YX, Huang YX. Clinical value of megakaryocytes in the diagnosis and treatment of children with immune thrombocytopenic purpura. Chin J Prim Med Pharm. 2019;26(23):2830-2834.

34. Chang TY. Modifying risk factors of chronicity in children with immune thrombocytopenia: still underway. Pediatr Neonatol. 2019;60(4):357-358. doi:10.1016/j.pedneo.2019.06.007

35. Del Vecchio GC, De Santis A, Giordano P, et al. Management of acute childhood idiopathic thrombocytopenic purpura according to AIEOP consensus guidelines: assessment of Italian experience. Acta Haematol. 2008;119(1):1-7. doi:10.1159/000112837

36. Ahmed I, Rajpurkar M, Thomas R, et al. Initial lymphocyte count and the development of persistent/chronic immune thrombocytopenic purpura. Pediatr Blood Cancer. 2010;55(3):508-511. doi:10.1002/ pbc. 22570

37. Gao CJ, Chen YE, Zhang Q, et al. Investigation of anxiety and depression in parents of children with immune thrombocytopenia. Pract Prev Med. 2017;24(3):337-339.

38. Zhou M, Xu Q. Research on quality of life in children with immune thrombocytopenia. J Appl Clin Pediatr. 2017;32(15):1132-1136.

39. Wu JX. Analysis of psychological behavior characteristics and influencing factors of children with chronic diseases. Womens Health Res. 2018;(9):104,186.

40. Guidry JA, George JN, Vesely SK, et al. Corticosteroid side-effects and risk for bleeding in immune thrombocytopenic purpura: patient and hematologist perspectives. Eur J Haematol. 2009;83(3):175-182. doi:10.1111/j.1600-0609.2009.01265.x

41. Giordano P, Lassandro G, Palladino V, et al. A narrative approach to describe QoL in children with chronic ITP. Front Pediatr. 2019;7:163. doi:10.3389/fped.2019.00163
International Journal of General Medicine

\section{Publish your work in this journal}

The International Journal of General Medicine is an international, peer-reviewed open-access journal that focuses on general and internal medicine, pathogenesis, epidemiology, diagnosis, monitoring and treatment protocols. The journal is characterized by the rapid reporting of reviews, original research and clinical studies across all disease areas. The manuscript management system is completely online and includes a very quick and fair peer-review system, which is all easy to use. Visit http://www.dovepress.com/ testimonials.php to read real quotes from published authors. 